\title{
SECTIONAL REPRESENTATION OF BANACH MODULES AND THEIR MULTIPLIERS
}

\author{
TERJE HÕIM and D. A. ROBBINS
}

Received 9 July 2002

\begin{abstract}
Let $X$ be a Banach module over the commutative Banach algebra $A$ with maximal ideal space $\Delta$. We show that there is a norm-decreasing representation of $X$, as a space of bounded sections in a Banach bundle $\pi: \mathscr{E} \rightarrow \Delta$, whose fibers are quotient modules of $X$. There is also a representation of $M(X)$, the space of multipliers $T: A \rightarrow X$, as a space of sections in the same bundle, but this representation may not be continuous. These sectional representations subsume results of various authors over the past three decades.
\end{abstract}

2000 Mathematics Subject Classification: 46H25, 46M20.

In this paper, $A$ will always be a commutative Banach algebra. Denote by $\Delta=\Delta_{A}$ the space of multiplicative functionals on $A$, and for $h \in \Delta$, let $K_{h}=$ ker $h \subset A$ be the corresponding maximal ideal. We give $\Delta$ its weak-* topology. Let $X$ be a Banach $A$-module and for $h \in \Delta$, let $K_{h} X$ be the closure in $X$ of $\operatorname{span}\left\{a x: a \in K_{h}, x \in X\right\}$. As usual, $C_{0}(\Delta)$ is the space of continuous complexvalued functions on $\Delta$ which vanish at infinity and ${ }^{\wedge}: A \rightarrow C_{0}(\Delta)$ is the Gelfand representation of $A$. Following the notation of Takahasi [9], if $h \in \Delta$, we choose $e_{h} \in A$ such that $\widehat{e_{h}}(h)=h\left(e_{h}\right)=1$, and we let $X^{h}$ be the closure in $X$ of $K_{h} X+\left(1-e_{h}\right) X$; it is easy to show that $X^{h}$ is independent of the choice of $e_{h}$. We set $X_{h}=X / X^{h}$. Denote by $X_{e}$ the essential part of $X$, that is, $X_{e}$ is the closed span of $\{a x: a \in A, x \in X\}$. If $X=X_{e}$, then $X^{h}=K_{h} X$. We denote by $M(X)$ the space of continuous multipliers $T: A \rightarrow X$, that is, the space of continuous $A$-module homomorphisms from $A$ to $X$. (So, if $T \in M(X)$, then $T(a b)=a T(b)$ for all $a, b \in A$.) If $x \in X$, denote by $T_{x}$ the multiplier defined by $T_{x}(a)=a x$.

We refer the reader to [1, 2, 3] for fundamental notions regarding bundles of Banach spaces and Banach modules. If $\pi: \mathscr{E} \rightarrow \Delta$ is a Banach bundle, we denote by $\mathscr{C}(\mathscr{E})$ (resp., $\mathscr{C}^{b}(\mathscr{E})$ ) the spaces of all (respectively, bounded) selections (= choice functions) $\sigma: \Delta \rightarrow \mathscr{E}$, by $\Gamma(\pi)$ the space of sections (= continuous choice functions) $\sigma: \Delta \rightarrow \mathscr{E}$, and by $\Gamma^{b}(\pi)$ and $\Gamma_{0}(\pi)$ the subspaces of $\Gamma(\pi)$ which, respectively, are bounded and vanish at infinity. We especially draw upon the following result, which is a special case of [2, Corollary 3.7].

Proposition 1 (see [3, Proposition 1.3]). Let $U$ be a topological space and let $\left\{X^{p}: p \in U\right\}$ be a collection of closed subspaces of the Banach space X. Let 
$\mathscr{E}=\dot{\bigcup}\left\{X / X^{p}: p \in U\right\}$ be the disjoint union of the quotient spaces $X / X^{p}$. Then, $\mathscr{E}$ can be topologized uniquely in such a way that the conditions (1) $\pi: \mathscr{E} \rightarrow U$ is a bundle of Banach spaces; and (2) for each $x \in X$, the selection $\tilde{x}: U \rightarrow \mathscr{E}$, $\tilde{x}(p)=x+X^{p}$ is a bounded section of the bundle $\pi: \mathscr{E} \rightarrow U$ are satisfied if and only if the function $p \mapsto\|\tilde{x}(p)\|$ is upper semicontinuous on $U$ for each $x \in X$.

Let $A$ be a commutative Banach algebra and let $X$ be a Banach $A$-module. Let $\mathscr{E}=\dot{\bigcup}\left\{X_{h}: h \in \Delta\right\}$ be the disjoint union of the $X_{h}$. We give an element $x+X^{h} \in X_{h} \subset \mathscr{E}$ its quotient norm $\left\|x+X^{h}\right\|$ and we let $\pi: \mathscr{E} \rightarrow \Delta$ be the obvious projection.

In [10], it was shown that if $\Delta$ is a compact Hausdorff space, then any (unital) module $X$ over $A=C(\Delta)$ can be represented as a space of sections in a bundle $\pi: \mathscr{E} \rightarrow \Delta$ of Banach spaces, in this case, with fibers $E_{h}=X_{h}=X / K_{h} X$. This (clearly norm-decreasing) representation is given by ${ }^{\sim}: X \rightarrow \Gamma(\pi), \tilde{x}(h)=$ $x+X^{h} \in X_{h}$, and satisfies the equation $\widetilde{a x}(h)=\hat{a}(h) \tilde{x}(h)$. In [3], it was shown that when $X$ is an essential module over a commutative algebra $A$ with bounded approximate identity, then there is a bundle $\pi: \mathscr{E} \rightarrow \Delta$, again, with fibers $E_{h}=X_{h}=X / K_{h} X$, and a (again, norm-decreasing) representation ${ }^{\sim}: X \rightarrow \Gamma_{0}(\pi)$ satisfying the same equation. Using the quotient modules suggested in [9], it was shown in [7] that when $A$ has a bounded approximate identity and $X$ is any Banach $A$-module, not necessarily essential, then there is in fact a bundle $\pi: \mathscr{E} \rightarrow \Delta$ with fibers $E_{h}=X_{h}=X / X^{h}$ and a norm-decreasing representation $\sim: M(X) \rightarrow \Gamma^{b}(\pi)$ given by $\tilde{T}(h)=T\left(e_{h}\right)+X^{h}$. Again, $\widetilde{a T}(h)=\hat{a}(h) \tilde{T}(h)$.

The purpose of this paper is to show that this notion of sectional representation can be extended to modules $X$ over arbitrary commutative Banach algebras $A$, that is, the earlier conditions that $X$ is essential or that $A$ have a bounded approximate identity can be removed. Thus, the representation obtained will be norm decreasing. We also show that $M(X)$ can be represented by sections in the same bundle as $X$, and give an example to show that this representation need not be continuous.

We define a map ${ }^{\sim}: X \rightarrow \mathscr{C}^{b}(\mathscr{E})$ by $\tilde{x}(h)=x+X^{h}$. We also define a map $\sim: M(X) \rightarrow \mathscr{C}(\mathscr{E})$, the space of all choice functions from $\Delta$ to $\mathscr{E}$, by $\tilde{T}(h)=$ $T\left(e_{h}\right)+X^{h}$. From a remark in [7], we note that the equations $\widetilde{a x}(h)=\hat{a}(h) \tilde{x}(h)$ and $\widetilde{a T}(h)=\hat{a}(h) \widetilde{T}(h)$ still hold. For $x \in X$ and for $h \in \Delta$, we have

$$
\widetilde{T_{x}}(h)=T_{x}\left(e_{h}\right)+X^{h}=e_{h} x+X^{h}=\widetilde{e_{h} x}(h)=\widehat{e_{h}}(h) \tilde{x}(h)=\tilde{x}(h),
$$

that is, $\tilde{x}=\widetilde{T_{x}}$.

We now demonstrate that the selections $\left\{\widetilde{T_{x}}: x \in X, \widetilde{T_{x}}(h)=e_{h} x+X^{h}=\right.$ $x+X^{h}$ for $\left.h \in \Delta\right\}$ generate a (unique) bundle topology on $\mathscr{E}=\dot{\bigcup}\left\{X_{h}: h \in \Delta\right\}$ in the most general situation (although the next proposition actually shows a little more than we need). 
Proposition 2. Let $A$ be a commutative Banach algebra, let $X$ be a Banach module over $A$, and let $T \in M(X)$. Then, the mapping $h \mapsto\|\widetilde{T}(h)\|=\| T\left(e_{h}\right)+$ $X^{h} \|$ is upper semicontinuous on $\Delta$.

Proof. The proof follows that of [7, Proposition 2.5] with only one alteration. Suppose that $\varepsilon>0$ is given and that $\|\tilde{T}(h)\|<\varepsilon$. Choose $a_{i} \in K_{h}, y_{i} \in X$, $(i=1, \ldots, n)$, and $z \in X$ such that

$$
\|\tilde{T}(h)\| \leq\left\|T\left(e_{h}\right)+\sum a_{i} y_{i}+\left(1-e_{h}\right) z\right\|<\varepsilon
$$

and set

$$
\varepsilon^{\prime}=\varepsilon-\left\|T\left(e_{h}\right)+\sum a_{i} y_{i}+\left(1-e_{h}\right) z\right\| .
$$

The functions $h^{\prime} \mapsto\left\|a_{i}+K_{h^{\prime}}\right\|$ are all upper semicontinuous on $\Delta$, the function $h^{\prime} \mapsto\left\|h^{\prime}\right\|$ is lower semicontinuous on $\Delta$, and $\left\|a+K_{h^{\prime}}\right\|=\left|\hat{a}\left(h^{\prime}\right)\right| /\left\|h^{\prime}\right\|$ (see [4]). (In particular, the lower semicontinuity of $h^{\prime} \mapsto\left\|h^{\prime}\right\|$ is what allows us to obtain the results in all what follows in this paper.) The function $\widehat{e_{h}}$ is also continuous on $\Delta$. We can therefore find a neighborhood $V$ of $h$ such that when $h^{\prime} \in V$, all of the following hold:

$$
\begin{gathered}
\sum\left\|a_{i}+K_{h^{\prime}}\right\|<\frac{\varepsilon^{\prime}}{3\left(\sum\left\|y_{i}\right\|+1\right)} ; \\
\left|\frac{1}{\widehat{e_{h}}(h)}-\frac{1}{\widehat{e_{h}}\left(h^{\prime}\right)}\right|=\left|1-\frac{1}{\widehat{e_{h}}\left(h^{\prime}\right)}\right|<\frac{\varepsilon^{\prime}}{3\left(\left\|T\left(e_{h}\right)\right\|+1\right)} ; \\
\left\|h^{\prime}\right\|>\frac{\|h\|}{2}>0 ; \quad\left|1-\widehat{e_{h}}\left(h^{\prime}\right)\right|<\frac{\varepsilon^{\prime}\|h\|}{6(\|z\|+1)} .
\end{gathered}
$$

Since the definition of $X^{h^{\prime}}$ is independent of the choice of $e_{h^{\prime}}$ for $h^{\prime} \in V$, we may as well take $e_{h^{\prime}}=\left(1 / \widehat{e_{h}}\left(h^{\prime}\right)\right) e_{h}$.

Then for $h^{\prime} \in V$, we have

$$
\begin{aligned}
\left\|\tilde{T}\left(h^{\prime}\right)\right\|= & \left\|T\left(e_{h^{\prime}}\right)+X^{h^{\prime}}\right\| \\
\leq & \left\|T\left(e_{h^{\prime}}\right)-T\left(e_{h}\right)+X^{h^{\prime}}\right\|+\left\|T\left(e_{h}\right)+\sum a_{i} y_{i}+\left(1-e_{h}\right) z+X^{h^{\prime}}\right\| \\
& +\left\|\sum a_{i} y_{i}+\left(1-e_{h}\right) z+X^{h^{\prime}}\right\| \\
\leq & \left\|T\left(e_{h^{\prime}}\right)-T\left(e_{h}\right)\right\|+\left\|T\left(e_{h}\right)+\sum a_{i} y_{i}+\left(1-e_{h}\right) z\right\| \\
& +\left\|\sum a_{i} y_{i}+X^{h^{\prime}}\right\|+\left\|\left(e_{h}-e_{h^{\prime}}\right) z+X^{h}\right\|
\end{aligned}
$$




$$
\begin{aligned}
&=\left|1-\widehat{e_{h}}\left(h^{\prime}\right)\right|\left\|T\left(e_{h}\right)\right\|+\left\|T\left(e_{h}\right)+\sum a_{i} y_{i}+\left(1-e_{h}\right) z\right\| \\
&+\left\|\sum a_{i} y_{i}+X^{h^{\prime}}\right\|+\left\|\left(e_{h}-e_{h^{\prime}}\right) z+X^{h^{\prime}}\right\| \\
& \leq \frac{\varepsilon^{\prime}}{3}+\left\|T\left(e_{h}\right)+\sum a_{i} y_{i}+\left(1-e_{h}\right) z\right\|+\left\|\sum a_{i} y_{i}+K_{h^{\prime}} X\right\| \\
&+\left\|\left(e_{h}-e_{h^{\prime}}\right)+K_{h^{\prime}}\right\|\left\|z+K_{h^{\prime}} X\right\| \\
& \leq \frac{\varepsilon^{\prime}}{3}+\left\|T\left(e_{h}\right)+\sum a_{i} y_{i}+\left(1-e_{h}\right) z\right\|+\sum\left\|a_{i}+K_{h^{\prime}} \mid\right\| y_{i} \| \\
&+\frac{\left|\widehat{e_{h^{\prime}}}\left(h^{\prime}\right)-\widehat{e_{h}}\left(h^{\prime}\right)\right|}{\left\|h^{\prime}\right\|}\|z\| \\
&<\varepsilon .
\end{aligned}
$$

Note that the key to the above generalization is the observation that the function $h^{\prime} \mapsto\left\|h^{\prime}\right\|$ is locally bounded away from 0 . This will also play a part in the sequel.

COROLlaRY 3. Let $A$ be a commutative Banach algebra and $X$ a Banach module over $A$. Then there is a unique topology on $\mathscr{E}=\dot{\bigcup}\left\{X_{h}: h \in \Delta\right\}$ which makes $\pi: \mathscr{E} \rightarrow \Delta$ a bundle of Banach spaces, and such that for each $x \in X$, $\tilde{x}: \Delta \rightarrow \mathscr{E}$ is an element of $\Gamma^{b}(\pi)$.

Proof. Note that for $x \in X$, we have $\widetilde{T_{x}}=\tilde{x}: \Delta \rightarrow \mathscr{E}$, and apply Propositions 1 and 2.

We call this mapping $\sim: X \rightarrow \Gamma(\pi)$ the Gelfand representation of $X$ and $\pi: \mathscr{E} \rightarrow \Delta$ the canonical bundle for $X$. (In [7], $\pi: \mathscr{E} \rightarrow \Delta$ is also called the multiplier bundle for $X$. The reason for this nomenclature shift has to do with the universal property that is to be discussed later.)

Recall now that in the bundle topology on $\mathscr{E}$, neighborhoods of a point $x+X^{h}$ are described by tubes. Let $\sigma \in \Gamma^{b}(\pi)$ be such that $\sigma(h)=x+X^{h}$, let $V$ be a neighborhood of $h$ in $\Delta$, and let $\varepsilon>0$. Then, $\mathscr{T}=\mathscr{T}(V, \sigma, \varepsilon)=\left\{z+X^{h^{\prime}}: h^{\prime} \in\right.$ $\left.V,\left\|\sigma\left(h^{\prime}\right)-\left(z+X^{h^{\prime}}\right)\right\|<\varepsilon\right\}$ is a neighborhood of $x+X^{h}$, and in fact sets of this form, as $V$ ranges over all neighborhoods of $h$ and $\varepsilon>0$ varies, form a fundamental system of neighborhoods of $\sigma(h)=x+X^{h}$. We rely on this description to prove the following corollary.

COROLlARY 4. Assume that $A$ and $X$ are as generally given and let $T \in$ $M(X)$. Then $\tilde{T} \in \Gamma(\pi)$.

Proof. Let $h \in \Delta$ be fixed, and set $\widetilde{T}(h)=x+X^{h}$ for some fixed $x \in X$. $\left(x=T\left(e_{h}\right) \in X\right.$ will do.) Let $\sigma \in \Gamma^{b}(\pi)$ be such that $\sigma(h)=x+X^{h}\left(\sigma=\widetilde{T\left(e_{h}\right)} \in\right.$ $\Gamma^{b}(\pi)$ will do.) Let $V$ be a neighborhood in $\Delta$ of $h$, and let $\varepsilon>0$. We need to 
find a neighborhood $V^{\prime}$ of $h$ such that $\tilde{T}\left(V^{\prime}\right) \subset \mathcal{T}(V, \sigma, \varepsilon)$. Since $\tilde{x} \in \Gamma^{b}(\pi)$ is continuous, there exists a neighborhood $V^{\prime} \subset V$ of $h$ such that if $h^{\prime} \in V^{\prime}$, then $\left\|\tilde{x}\left(h^{\prime}\right)-\sigma\left(h^{\prime}\right)\right\|<\varepsilon / 2$. Since $T-T_{x}$ is a multiplier of $X$, the map $h^{\prime} \mapsto$ $\left\|\left(\widetilde{T-T_{X}}\right)\left(h^{\prime}\right)\right\|$ is upper semicontinuous on $\Delta$, by Proposition 2 . Hence, there is a neighborhood $V^{\prime \prime} \subset V^{\prime}$ of $h$ such that if $h^{\prime} \in V^{\prime \prime}$, then by our choice of $x$, we have $\left\|\left(\widetilde{T-T_{x}}\right)\left(h^{\prime}\right)\right\|<\varepsilon / 2$ since $\left(\widetilde{T-T_{x}}\right)(h)=0$. Then, it follows immediately that for $h^{\prime} \in V^{\prime \prime}$, we have

$$
\left\|\tilde{T}\left(h^{\prime}\right)-\sigma\left(h^{\prime}\right)\right\| \leq\left\|\left(\widetilde{T-T_{x}}\right)\left(h^{\prime}\right)\right\|+\left\|\tilde{x}\left(h^{\prime}\right)-\sigma\left(h^{\prime}\right)\right\|<\varepsilon
$$

so that $\tilde{T}\left(h^{\prime}\right) \in \mathscr{T}(V, \sigma, \varepsilon)$.

In particular, if $T \in M(X)$, then $\tilde{T}$ is "locally close" in $\Gamma(\pi)$ to sections of the form $\widetilde{T_{x}}=\tilde{x}$.

It was shown in [7] that if $A$ has a bounded approximate identity and if $T \in M(X)$, then in fact $\widetilde{T}$ is bounded. In the absence of a bounded approximate identity, the boundedness of $\tilde{T}$ cannot be guaranteed, as in the next example.

EXAMPLE 5. For each $n \in \mathbb{N}$, let $E_{n}=\mathbb{C}$, with norm $\|\alpha\|_{n}=n|\alpha|$, and let $A=\left\{x: \mathbb{N} \rightarrow \mathbb{C}: \lim _{n}\|x(n)\|_{n}=0\right\}$. Then $A$ is a Banach algebra under the pointwise operations and norm $\|x\|=\sup _{n}\left\{\|x(n)\|_{n}\right\}$, and by [5], we have $\Delta=$ $\Delta_{A}=\left\{\phi_{n}: n \in \mathbb{N}\right\}$ where $\phi_{n}(x)=x(n)$. We have $\left\|\phi_{n}\right\|=\sup _{\|x\|=1}\left\{\left|\phi_{n}(x)\right|\right\}=$ $\sup _{\|x\|=1}\{|x(n)|\}$. If $\|x\|=1$, then for each $n$, we have $|x(n)| \leq 1 / n$; on the other hand, if $e_{n}$ is the standard basis vector $\left(e_{n}(j)=\delta_{n j}\right)$, then $\phi_{n}\left((1 / n) e_{n}\right)$ $=1 / n$ and $\left\|(1 / n) e_{n}\right\|=1$. Hence, $\left\|\phi_{n}\right\|=1 / n$, and so by [7, Lemma 2.3], $A$ has no bounded approximate identity (although $\left\{\sum_{k=1}^{n} e_{k}: n \in \mathbb{N}\right\}$ does form an approximate identity). Consider $A$ as a module over itself. The sequence $y$ given by $y(n)=1 / \sqrt{n}$ defines a multiplier $T_{y}$ on $A, T_{y}(x)(n)=x(n) y(n)=$ $(1 / \sqrt{n}) x(n)$, and it is easy to see that $T_{y}$ is norm decreasing. Note that $y \notin A$ since $\|y\|=\infty$. For each $n,\left\|\widetilde{T_{y}}(n)\right\|=\left\|T_{y}\left(e_{n}\right)+A^{\phi_{n}}\right\|=\inf \left\{\left\|(1 / \sqrt{n}) e_{n}+a\right\|\right.$ : $\left.a \in A^{\phi_{n}}\right\}$. Now, $A^{\phi_{n}}=\operatorname{ker} \phi_{n}=\{a \in A: a(n)=0\}$ and so

$$
\inf \left\{\left\|\frac{1}{\sqrt{n}} e_{n}+a\right\|: a \in A^{\phi_{n}}\right\}=\inf \left\{\left\|\frac{1}{\sqrt{n}} e_{n}\right\|+\|a\|: a \in A^{\phi_{n}}\right\}=\sqrt{n} .
$$

Thus, $\widetilde{T_{y}}$ is unbounded.

Moreover, if $T \in M(A)$ and if $\tilde{T}$ is bounded, then $T=T_{y}$ for some $y \in A$. To see this, let $n, k \in \mathbb{N}$ and let $T \in M(A)$. Then $\left(e_{n} T\left(e_{n}\right)\right)(k)=e_{n}(k) T e_{n}(k)=$ $\delta_{n k} T e_{n}(k)=0$ if $n \neq k$. Define $y$ by $y(n)=\left(T e_{n}\right)(n)$; clearly, for $a \in A$, we have

$$
T(a)(n)=e_{n}(n)(T a)(n)=a(n)\left(T e_{n}\right)(n)=a(n) y(n)=\left(T_{y} a\right)(n),
$$

so that $T=T_{y}$. 
Now, suppose that $\widetilde{T}=\widetilde{T_{y}}$ is bounded; we will show that $y \in A$. In fact, for all $n$, we have

$$
\begin{aligned}
\|y(n)\|_{n} & =\left\|y(n) e_{n}(n)\right\|_{n}=\left\|T_{y}\left(e_{n}\right)\right\| \\
& =\inf \left\{\left\|T_{y}\left(e_{n}\right)+a\right\|: a \in A^{\phi_{n}}\right\}=\left\|\widetilde{T_{y}}\left(\phi_{n}\right)\right\| \\
& \leq\left\|T_{y}\right\|\left\|\phi_{n}\right\|=\frac{\left\|T_{y}\right\|}{n} \longrightarrow 0
\end{aligned}
$$

as $n \rightarrow \infty$. Hence, $y \in A$.

On the basis of this example, we ask the following: let $T \in M(X)$. If $\tilde{T} \in$ $\Gamma^{b}(\pi)$, what conditions on $A$ and $X$ will guarantee that there is some $x \in X$ such that $\widetilde{T_{x}}=\tilde{x}$ ? If $\tilde{T} \in \Gamma_{0}(\pi)$, what conditions on $A$ and $X$ will guarantee that $\widetilde{T_{x}}=\tilde{x}$ for some $x \in X_{e}$ ?

Now, let $A$ and $X$ be as generally given. Following [3, Section 2], if $\Psi: X \rightarrow$ $\Gamma(\rho)$ is a bounded map, where $\rho: \mathscr{F} \rightarrow \Delta=\Delta_{A}$ is a Banach bundle, we will call $\Psi$ a sectional representation of Gelfand type provided that $\Psi(a x)=\hat{a} \Psi(x)$. In [3, Theorem 2.7], it is shown that if $A$ has a bounded approximate identity and if $X$ is an essential $A$-module, then the representation ${ }^{\sim}: X \rightarrow \Gamma_{0}(\pi)$, where $\pi: \mathscr{E} \rightarrow \Delta$ is the canonical bundle for $X$ described above, is universal with respect to all sectional representations of $X$ of Gelfand type. In that context, this means that if $\rho: \mathscr{F} \rightarrow \Delta$ is a bundle of Banach spaces and if $\Psi: X \rightarrow \Gamma_{0}(\rho)$ is a sectional representation of Gelfand type, then there is a unique continuous map $\tilde{\Psi}: \mathscr{E} \rightarrow \mathscr{F}$ which is fiber-preserving (meaning $\tilde{\Psi}\left(E_{h}\right) \subset F_{h}$ ) and linear on each fiber. Moreover, $\|\tilde{\Psi}\|=\sup _{h}\left\{\left\|\widetilde{\Psi} \mid E_{h}\right\|\right\} \leq\|\Psi\|$ and $\Psi(x)=\tilde{\Psi} \circ \tilde{x}$. When $A$ has a bounded approximate identity and $X$ is essential, this universal property characterizes the canonical bundle $\pi: \mathscr{E} \rightarrow \Delta$ up to isomorphism. The same universal property can now be shown to obtain in the general case.

Proposition 6. Let $A$ and $X$ be as generally given and let $\pi: \mathscr{E} \rightarrow \Delta$ be the canonical bundle for $X$ constructed in Corollary 3. Let $\rho: F \rightarrow \Delta$ be a Banach bundle with fibers $\left\{F_{h}: h \in \Delta\right\}$ and suppose that $\Psi: X \rightarrow \Gamma(\rho)$ is a sectional representation of Gelfand type. Then, there exists a unique fiber-preserving continuous map $\tilde{\Psi}: \mathscr{E} \rightarrow \mathscr{F}$ such that $\|\tilde{\Psi}\| \leq\|\Psi\|$ and such that $\Psi(x)=\tilde{\Psi} \circ \tilde{x}$.

Proof. For $h \in \Delta$, define $\Psi_{h}: X \rightarrow F_{h}$ by $\Psi_{h}(x)=[\Psi(x)](h)$. Then the kernel of $\Psi_{h}$ contains $X^{h}$ (Justification: $\Psi_{h}\left(a x+\left(1-e_{h}\right) z\right)=\left[\Psi\left(a x+\left(1-e_{h}\right) z\right)\right](h)=$ 0 for $a \in K_{h}$ and $x, z \in X$, because $\Psi$ is of Gelfand type and because $\left.\widehat{e_{h}}(h)=1\right)$. This induces a map $\widetilde{\Psi_{h}}: X_{h}=X / X^{h} \rightarrow F_{h}$ such that $\widetilde{\Psi_{h}}\left(x+X^{h}\right)=\Psi_{h}(x)=$ $[\Psi(x)](h)$, and we define $\widetilde{\Psi}$ on all of $\mathscr{E}$ by $\widetilde{\Psi}\left(x+X^{h}\right)=\widetilde{\Psi_{h}}\left(x+X^{h}\right)$. Clearly, $\|\widetilde{\Psi}\|=\sup \left\{\left\|\widetilde{\Psi_{h}}\right\|: h \in \Delta\right\} \leq\|\Psi\|$. We can now essentially repeat the proof of [3, Theorem 2.7] to get the desired result. 
COROLLARY 7. Let $A$ and $X$ be as generally given. Suppose that $\rho_{k}: \mathscr{F}_{k} \rightarrow \Delta$ are Banach bundles and that $\phi_{k}: X \rightarrow \Gamma\left(\rho_{k}\right)(k=1,2)$ are Gelfand representations of $X$. Suppose also that if $\phi: X \rightarrow \Gamma(\xi)(\xi: G \rightarrow \Delta)$ is any sectional representation of Gelfand type, then there exist unique continuous, fiber-preserving, and linear-on-fibers maps $\widetilde{\phi_{k}}: \mathscr{F}_{k} \rightarrow \varphi$ such that $\left\|\widetilde{\phi_{k}}\right\| \leq\|\phi\|$ and such that $\phi(x)=\widetilde{\phi}_{k} \circ \phi_{k}(x)(k=1,2)$. Then, there exists a continuous map $\Phi: \mathscr{F}_{1} \rightarrow \mathscr{F}_{2}$ such that $\phi_{2}(x)=\Phi \circ \phi_{1}(x)$ for all $x \in X$, and such that $\Phi$ is fiber-preserving and a linear isomorphism on each fiber.

Proof. Here, we may repeat the proof of [3, Proposition 2.8].

In [7, Proposition 2.8], it is shown that in the presence of a bounded approximate identity in $A$, the canonical bundles $\pi: \mathscr{E} \rightarrow \Delta$ for $X$ and $\pi^{\prime}: \mathscr{E}^{\prime} \rightarrow \Delta$ for $X_{e}$ are homeomorphic. The proof uses the facts that (1) with or without a bounded approximate identity in $A$, for $h \in \Delta, X_{h}$ and $X_{h}^{\prime}=X_{e} /\left(X_{e}\right)^{h}$ are topologically isomorphic via the maps $\psi_{h}: X_{h} \rightarrow X_{h}^{\prime}, \psi_{h}\left(x+X^{h}\right)=e_{h} x+K_{h} X_{e}$, and $\phi_{h}: X_{h}^{\prime} \rightarrow X_{h}, \phi_{h}\left(a x+K_{h} X_{e}\right)=a x+X^{h}$, with $\left\|\psi_{h}\right\| \leq\left\|e_{h}\right\|$ and $\left\|\phi_{h}\right\| \leq 1[7$, Proposition 2.7]; and (2) when $A$ has a bounded approximate identity, the set $S=\left\{e_{h}: h \in \Delta\right\}$ can be chosen to be bounded. The bound on $S$ is then used to obtain the homeomorphism. Even without the bounded approximate identity, we can easily modify the proof of [7, Proposition 2.8] to obtain the following proposition.

Proposition 8. Let $A$ and $X$ be as generally given and let $\pi: \mathscr{E} \rightarrow \Delta$ and $\pi^{\prime}: \mathscr{E}^{\prime} \rightarrow \Delta$ be the canonical bundles for $X$ and $X_{e}$, respectively. Then, $\mathscr{E}$ and $\mathscr{E}^{\prime}$ are homeomorphic in their bundle topologies.

Proof. We will show that the map $\Psi: \mathscr{E} \rightarrow \mathscr{E}^{\prime}$ given by $\Psi\left(x+X^{h}\right)=\psi_{h}(x+$ $\left.X^{h}\right)=e_{h} x+K_{h} X_{e}$ is continuous; the proof of the continuity of the inverse map $\Phi: \mathscr{E}^{\prime} \rightarrow \mathscr{E}, \Phi\left(a x+K_{h} X_{e}\right)=\phi_{h}\left(a x+K_{h} X_{e}\right)$ will be similar.

Fix $h \in \Delta$ and let $x+X^{h} \in \mathscr{E}$. Let $\mathscr{T}_{1}=\mathscr{T}_{1}\left(V, \widetilde{e_{h} x}, \varepsilon\right)$ be a tube around $e_{h} x+$ $K_{h} X_{e}=\Psi\left(x+X^{h}\right)=\psi_{h}\left(x+X^{h}\right) \in \mathscr{E}^{\prime}$, and let $V^{\prime} \subset V$ be a neighborhood of $h$ such that for $h^{\prime} \in V^{\prime}$, we have $\left|\widehat{e_{h}}\left(h^{\prime}\right)\right|>1 / 2$. In $V^{\prime}$, set $e_{h^{\prime}}=\left(1 / \widehat{e_{h}}\left(h^{\prime}\right)\right) e_{h}$; then $\left\|e_{h^{\prime}}\right\|<2\left\|e_{h}\right\|$. Then, $\mathscr{T}_{2}=\mathscr{T}_{2}\left(V^{\prime}, \tilde{x}, \varepsilon /\left(2\left\|e_{h}\right\|\right)\right)$ is a neighborhood of $x+X^{h}=$ $\tilde{x}(h)$ in $\mathscr{E}$. Taking $y+X^{h^{\prime}} \in \mathcal{T}_{2}$, we have $h^{\prime} \in V^{\prime}$ and

$$
\left\|\left(y+X^{h^{\prime}}\right)-\tilde{x}\left(h^{\prime}\right)\right\|=\left\|\left(y+X^{h^{\prime}}\right)-\left(x+X^{h^{\prime}}\right)\right\|<\frac{\varepsilon}{2\left\|e_{h}\right\|} .
$$

Then

$$
\begin{aligned}
\left\|\Psi\left(y+X^{h^{\prime}}\right)-\Psi\left(x+X^{h^{\prime}}\right)\right\| & =\left\|\psi_{h^{\prime}}\left(y+X^{h^{\prime}}\right)-\psi_{h^{\prime}}\left(x+X^{h^{\prime}}\right)\right\| \\
& \leq\left\|e_{h^{\prime}}\right\|\|\|\left(y+X^{h^{\prime}}\right)-\left(x+X^{h^{\prime}}\right) \|<\varepsilon
\end{aligned}
$$

so that $\Psi\left(y+X^{h^{\prime}}\right) \in \mathscr{T}_{1}$. 
We noted above that in the most general case, elements of the form $\tilde{T}$ ( $T \in$ $M(X))$ are locally close to elements of the form $\tilde{x}(x \in X)$. With an additional assumption on $A$, local closeness can be replaced by local equality.

Proposition 9. Let $A$ be a completely regular algebra and let $X$ be an $A$ module. Let $h \in \Delta$ and let $T \in M(X)$. Then for each compact set $V \subset \Delta$ containing $h$, there exists $x \in X_{e}$ such that $\tilde{T}\left(h^{\prime}\right)=\tilde{x}\left(h^{\prime}\right)$ for $h^{\prime} \in V$.

Proof. Let $V$ be such a compact set containing $h$. It then follows from [6, Theorem 2.7.12] that there exists $e \in A$ such that $\hat{e} \equiv 1$ on $V$. Set $x=e T(e) \in$ $X_{e}$, and for $h^{\prime} \in V$, let $e_{h^{\prime}}=e$. Then for $h^{\prime} \in V$, we have

$$
\widetilde{T}\left(h^{\prime}\right)=T\left(e_{h^{\prime}}\right)+X^{h^{\prime}}=T(e)+X^{h^{\prime}}=e T(e)+X^{h^{\prime}}=\widetilde{e T(e)}\left(h^{\prime}\right)=\tilde{x}\left(h^{\prime}\right) .
$$

Compare this to [8, Theorem 4.1 and Corollary 4.2]. In our case, for $T \in$ $M(X)$, it cannot be guaranteed that there might exist $x \in X$ such that $\tilde{T}=\tilde{x}$ on a neighborhood of infinity, because $\tilde{T}$ can be unbounded, while $\tilde{x}$ is bounded. However, in the event that there actually does exist $x \in X$ such that $\tilde{T}=\tilde{x}$ on some neighborhood of infinity, we obtain the following corollary, which is similar to [8, Corollary 4.2].

COROLlARY 10. Suppose that $A$ is completely regular and that $T \in M(X)$. If there exists a neighborhood $V$ of infinity such that for some $x \in X$, we have $\tilde{T}\left(h^{\prime}\right)=\tilde{x}\left(h^{\prime}\right)$ for $h^{\prime} \in V$, then there exists $y \in X$ such that $\tilde{T}=\tilde{y}$ on all of $\Delta$.

Proof. We can repeat the partition of unity proof of [8, Theorem 4.1].

ACKNOWLEDGMENT. The authors thank the referee for his careful reading of the manuscript.

\section{REFERENCES}

[1] G. Gierz, Bundles of Topological Vector Spaces and Their Duality, Lecture Notes in Mathematics, vol. 955, Springer-Verlag, Berlin, 1982.

[2] K. H. Hofmann, Bundles and sheaves are equivalent in the category of vector spaces, preprint, Tulane University, 1974.

[3] J. W. Kitchen and D. A. Robbins, Gelfand representation of Banach modules, Dissertationes Math. (Rozprawy Mat.) 203 (1982), 47.

[4] - Sectional representations of Banach modules, Pacific J. Math. 109 (1983), no. $1,135-156$.

[5] _ _ Bundles of Banach algebras. II, Houston J. Math. 20 (1994), no. 3, 435451.

[6] C. E. Rickart, General Theory of Banach Algebras, The University Series in Higher Mathematics, D. Van Nostrand, New Jersey, 1960.

[7] D. A. Robbins, BSE Banach modules and bundles of Banach spaces, Houston J. Math. 24 (1998), no. 3, 489-505.

[8] S.-E. Takahasi, An extension of Helson-Edwards theorem to Banach modules, Int. J. Math. Math. Sci. 14 (1991), no. 2, 227-232. 
[9] _ BSE Banach modules and multipliers, J. Funct. Anal. 125 (1994), no. 1, 67-89.

[10] J. Varela, Sectional representation of Banach modules, Math. Z. 139 (1974), 5561.

Terje Hõim: Department of Mathematics, Trinity College, Hartford, CT 06106-3100, USA

E-mail address: terje.hoim@trincol1.edu

D. A. Robbins: Department of Mathematics, Trinity College, Hartford, CT 06106-3100, USA

E-mail address: david.robbins@trinco11.edu 


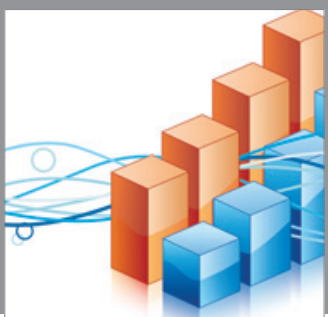

Advances in

Operations Research

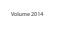

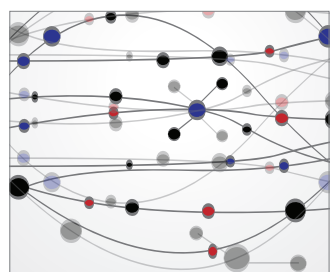

\section{The Scientific} World Journal
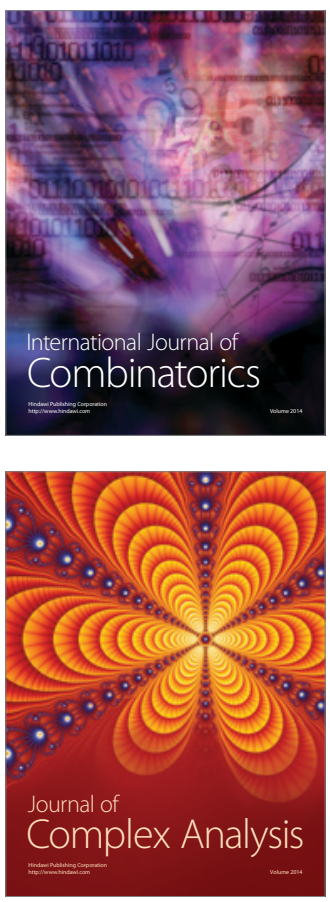

International Journal of

Mathematics and

Mathematical

Sciences
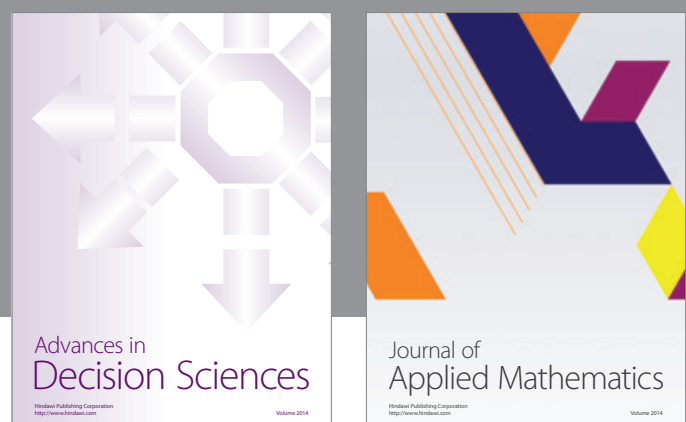

Journal of

Applied Mathematics
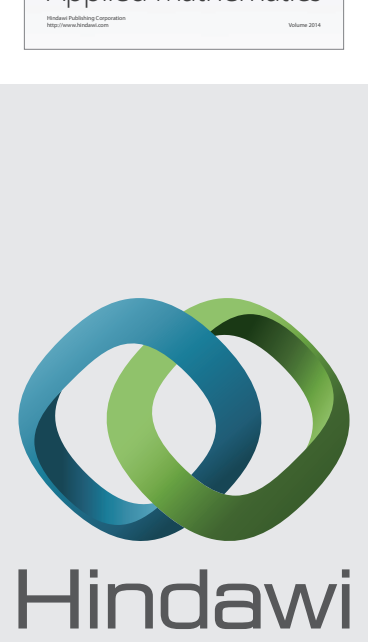

Submit your manuscripts at http://www.hindawi.com
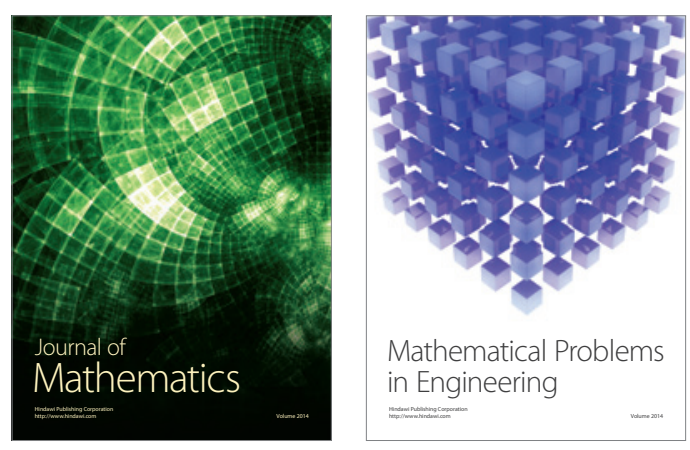

Mathematical Problems in Engineering
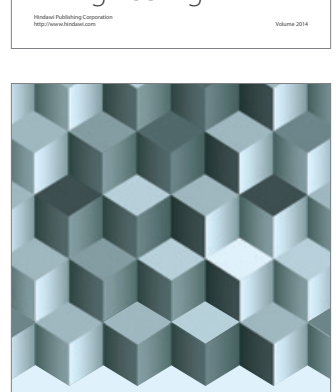

Journal of

Function Spaces
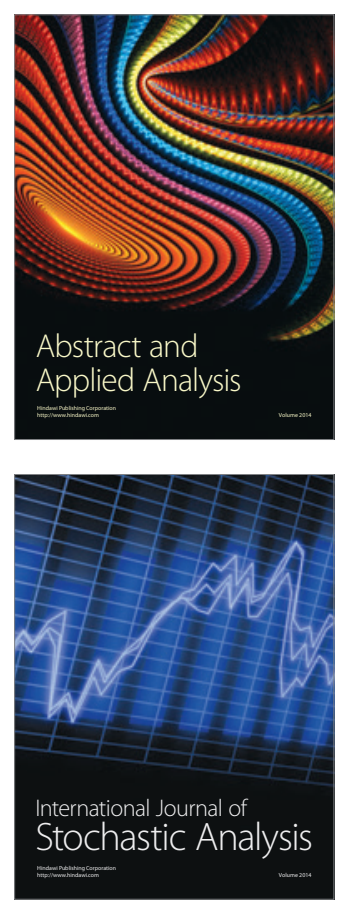

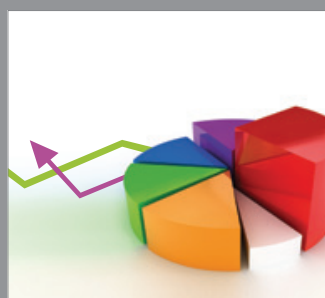

ournal of

Probability and Statistics

Promensencen
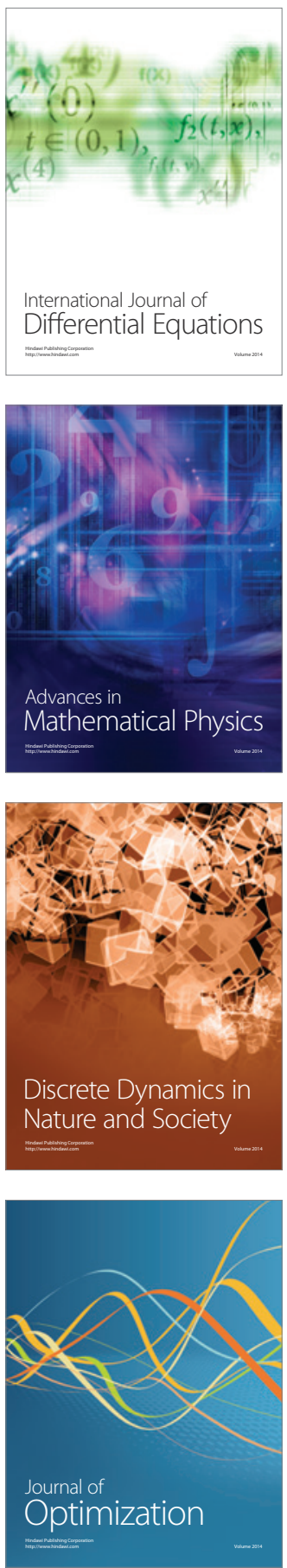